\title{
Synthesis, spectral characterization and biological evaluation of 4H-1,4-benzothiazines, their sulfones and ribofuranosides
}

\author{
Naveen Gautam, Neha Ajmera*, Shikha Gupta and Dinesh Chand Gautam \\ Department of Chemistry, University of Rajasthan, Jaipur-302004, India \\ ${ }^{*}$ Corresponding author at: Department of Chemistry, University of Rajasthan, Jaipur-302004, India. Tel.: +91.992.8537300; fax: +91.141.4004930 \\ E-mail address: ajmneha@yahoo.com (N. Ajmera).
}

\section{ARTICLE INFORMATION}

Received: 16 July 2011

Received in revised form: 04 September 2011

Accepted: 23 September 2011

Online: 31 March 2012

\section{KEYWORDS}

Sulfones

Heterocycles

Ribofuranosides

Antioxidant activity

Antimicrobial activity

4H-1,4-benzothiazines

\begin{abstract}
Synthesis of heterocyclic compounds like benzothiazines has attracted attention in recent years due to their biological and industrial value. This article reflects up-to-date and comprehensive coverage of biochemical aspects of benzothiazines, their sulfones and ribofuranosides. The nitrogen and sulfur containing heterocycles were prepared by condensation followed by oxidative cyclization of 2 -aminobenzenethiol with $\beta$-diketones/ $\beta$ ketoesters in dimethylsulfoxide. These compounds were then used as base to prepare ribofuranosides by treating them with sugar ( $\beta$-D-ribofuranose-1-acetate-2,3,5-tribenzoate). On refluxing with hydrogen peroxide in glacial acetic acid, these substituted dimethyl $4 H-1,4-$ benzothiazines yielded 4H-1,4-benzothiazine-1,1-dioxides. Antioxidant and antimicrobial activity of these compounds were carried out and structure evaluation was done by spectral and elemental analysis.
\end{abstract}

\section{Introduction}

The synthesis of benzothiazines, their sulfones and ribofuranosides has attracted tremendous interest due to wide spectrum of biological activities possessed by these compounds such as antibacterial, CNS depressants, antichloesterolic, anticancer, antifungal etc. As a part of the ongoing study, we have synthesized some new benzothiazines, their sulfones and ribofuranosides. Substituted benzothiazines were prepared by condensation of 2 -aminobenzenethiols with $\beta$-diketone $/ \beta$ ketoester in presence of dimethylsulfoxide through oxidative cyclization. Intermediate bis-(2-aminophenyl)disulfides undergoes cyclization through scission of $\mathrm{S}-\mathrm{S}$ bond due to high reactivity of alpha position of enaminoketone system towards nucleophilic attack. The structures of these compounds were determined on the basis of spectral data and elemental analysis. These compounds were also screened for biological activity [1-11].

\section{Experimental}

All the melting points were determined in open capillary tubes but are uncorrected. ${ }^{1} \mathrm{H}$ NMR and ${ }^{13} \mathrm{C}$ NMR were recorded on JEOL AL 300 spectrometer $(300 \mathrm{MHz})$ in DMSO- $d_{6} / \mathrm{CDCl}_{3}$ using TMS (tetramethyl silane) as an internal standard (Chemical shifts are measured in $\delta$, ppm). IR spectra were recorded in $\mathrm{KBr}$ on SHIMADZU $8400 \mathrm{~S}$ FTIR spectrophotometer. Mass spectra were recorded on JEOL SX 102/DA 600 using Xenon/Argon as FAB (Fast Atom Bombardment) gas. The purity of compounds were checked by thin layer chromatography using silica gel "G" as adsorbent, visualizing these by UV light or in an Iodine chamber. Elemental analysis of these compounds was also done.

\subsection{General method of synthesis of substituted $4 \mathrm{H}-1,4-$ benzothiazine (3a-d)}

To a stirred suspension of 0.01 mole of $\beta$-diketone $/ \beta$ ketoester (2a-d) in $5 \mathrm{~mL}$ of dimethylsulfoxide was added 0.01 mole of 2-aminobenzenethiol (1) and resulting mixture was refluxed for 20 minutes (Table 1). The reaction mixture was concentrated, cooled down to room temperature. The solid separated out was filtered, washed with petroleum ether and crystallized from methanol (Scheme 1). Characterization data and spectral analysis of each compound (3a-d) is given as:

Ethyl-3-trifluoromethyl-6,8-dimethyl-4H-1,4-benzothiazine2-carboxylate (3a): Yield: $46 \%$. M.p.: $55{ }^{\circ} \mathrm{C}$. IR $\left(\mathrm{KBr}, v, \mathrm{~cm}^{-1}\right)$ : 3260 (N-H), 1690 (>C=0), 1340, 1160 ( $\mathrm{CF}_{3}$ str.), 1255, 1015 (CO-C str.), 2885 ( $-\mathrm{CH}_{3}$ str.). ${ }^{1} \mathrm{H}$ NMR (DMSO- $d_{6}, 300 \mathrm{MHz}, \delta, \mathrm{ppm}$ ): $8.90(\mathrm{~s}, 1 \mathrm{H}, \mathrm{N}-\mathrm{H}), 8.08-7.20(\mathrm{~m}, 2 \mathrm{H}$, aromatic-H), $4.19(\mathrm{q}, 2 \mathrm{H}, J=$ $6.9 \mathrm{~Hz}, \mathrm{CH}_{2}$ of $\mathrm{C}_{2} \mathrm{H}_{5}$ at $\left.\mathrm{C}_{2}\right), 1.30\left(\mathrm{t}, 3 \mathrm{H}, J=6.2 \mathrm{~Hz}, \mathrm{CH}_{3}\right.$ of $\mathrm{C}_{2} \mathrm{H}_{5}$ at $\left.\mathrm{C}_{2}\right), 2.35\left(\mathrm{~s}, 3 \mathrm{H}, \mathrm{CH}_{3}\right.$ at $\left.\mathrm{C}_{6}\right), 2.36\left(\mathrm{~s}, 3 \mathrm{H}, \mathrm{CH}_{3}\right.$ at $\left.\mathrm{C}_{8}\right) .{ }^{13} \mathrm{C}$ NMR $\left(\mathrm{CDCl}_{3}, 300 \mathrm{MHz}, \delta, \mathrm{ppm}\right): 107.2(\mathrm{C}-2), 138.6$ (C-3), 113.1 (C-5), 135.2 (C-6), 120.2 (C-7), 139.1 (C-8), $21.2\left(\mathrm{CH}_{3}\right.$ at $\left.\mathrm{C}_{6}\right), 14.1\left(\mathrm{CH}_{3}\right.$ at $\left.\mathrm{C}_{8}\right), 165\left(\mathrm{C}\right.$ of $\mathrm{CO}$ at $\left.\mathrm{C}_{2}\right), 114\left(-\mathrm{CF}_{3}\right.$ at $\left.\mathrm{C}_{3}\right), 59.2\left(\mathrm{CH}_{2}\right.$ of $\mathrm{COOC}_{2} \mathrm{H}_{5}$ at $\left.\mathrm{C}_{2}\right), 13.8\left(\mathrm{CH}_{3}\right.$ of $\mathrm{COOC}_{2} \mathrm{H}_{5}$ at $\left.\mathrm{C}_{2}\right)$. MS $(\mathrm{m} / \mathrm{z}, \%): 317$ $\left(\mathrm{M}^{+}\right), 244$ (52), 202 (38), 275 (76), 73 (100). Anal. calcd. for $\mathrm{C}_{14} \mathrm{H}_{14} \mathrm{NO}_{2} \mathrm{~F}_{3} \mathrm{~S}$ : C, 52.99; $\mathrm{H}, 4.41$; N, 4.41. Found: $\mathrm{C}, 53.25 ; \mathrm{H}$, $4.39 ; \mathrm{N}, 4.35 \%$.

2-Trifluoroacetyl-3-trifluoromethyl-6,8-dimethyl-4H-1,4benzothiazine (3b): Yield: $68 \%$. M.p.: $65{ }^{\circ} \mathrm{C}$. IR $\left(\mathrm{KBr}, v, \mathrm{~cm}^{-1}\right)$ : $3385(\mathrm{~N}-\mathrm{H}), 1650(>\mathrm{C}=0), 1350,1180\left(\mathrm{CF}_{3} \mathrm{str}.\right), 2895\left(-\mathrm{CH}_{3}\right.$ str.). ${ }^{1} \mathrm{H}$ NMR (DMSO- $\left.d_{6}, 300 \mathrm{MHz}, \delta, \mathrm{ppm}\right): 8.29$ (s, $1 \mathrm{H}, \mathrm{NH}$ ), 8.26-6.06 (m, $2 \mathrm{H}$, aromatic- $\mathrm{H}), 2.35\left(\mathrm{~s}, 3 \mathrm{H}, \mathrm{CH}_{3}\right.$ at $\left.\mathrm{C}_{6}\right), 2.36(\mathrm{~s}$, $3 \mathrm{H},-\mathrm{CH}_{3}$ at $\left.\mathrm{C}_{8}\right) .{ }^{13} \mathrm{C} \mathrm{NMR}\left(\mathrm{CDCl}_{3}, 300 \mathrm{MHz}, \delta, \mathrm{ppm}\right): 116.2$ (C-2), 136.3 (C-3), 112.2 (C-5), 136.4 (C-6), 119.2 (C-7), 138.2 (C-8), $15.8\left(\mathrm{CH}_{3}\right.$ at $\left.\mathrm{C}_{6}\right), 20.1\left(\mathrm{CH}_{3}\right.$ at $\left.\mathrm{C}_{8}\right), 196.5\left(\mathrm{C}\right.$ of $\mathrm{CO}$ at $\left.\mathrm{C}_{2}\right), 129.8$ $\left(\mathrm{CF}_{3}\right.$ at $\left.\mathrm{C}_{2}\right), 114.1\left(\mathrm{CF}_{3}\right.$ at $\left.\mathrm{C}_{3}\right)$.

European Journal of Chemistry 
Table 1. The exact reaction times and yields of individual reactions (3a-d, $\mathbf{4 a - d}$, 5a-b)

\begin{tabular}{|c|c|c|c|c|}
\hline Compound No & $\mathbf{R}_{\mathbf{3}}$ & $\mathbf{R}_{4}$ & Reaction times (min.) & Yield (\%) \\
\hline $3 a$ & $\mathrm{CF}_{3}$ & $\mathrm{OC}_{2} \mathrm{H}_{5}$ & 22 & 46 \\
\hline $3 b$ & $\mathrm{CF}_{3}$ & $\mathrm{CF}_{3}$ & 20 & 68 \\
\hline $3 c$ & $\mathrm{CH}_{2} \mathrm{CH}_{3}$ & $\mathrm{OCH}_{3}$ & 23 & 76 \\
\hline $3 d$ & $\mathrm{CH}_{3}$ & $\mathrm{C}_{6} \mathrm{H}_{3}\left(\mathrm{OCH}_{3}\right)_{2}(o, p)$ & 21 & 80 \\
\hline $4 a$ & $\mathrm{CF}_{3}$ & $\mathrm{OC}_{2} \mathrm{H}_{5}$ & 255 & 69 \\
\hline $4 b$ & $\mathrm{CF}_{3}$ & $\mathrm{CF}_{3}$ & 275 & 72 \\
\hline $4 c$ & $\mathrm{CH}_{2} \mathrm{CH}_{3}$ & $\mathrm{OCH}_{3}$ & 295 & 56 \\
\hline $4 \mathrm{~d}$ & $\mathrm{CH}_{3}$ & $\mathrm{C}_{6} \mathrm{H}_{3}\left(\mathrm{OCH}_{3}\right)_{2}(o, p)$ & 260 & 82 \\
\hline $5 a$ & $\mathrm{CF}_{3}$ & $\mathrm{OC}_{2} \mathrm{H}_{5}$ & 920 & 84 \\
\hline $5 b$ & $\mathrm{CF}_{3}$ & $\mathrm{CF}_{3}$ & 980 & 68 \\
\hline
\end{tabular}
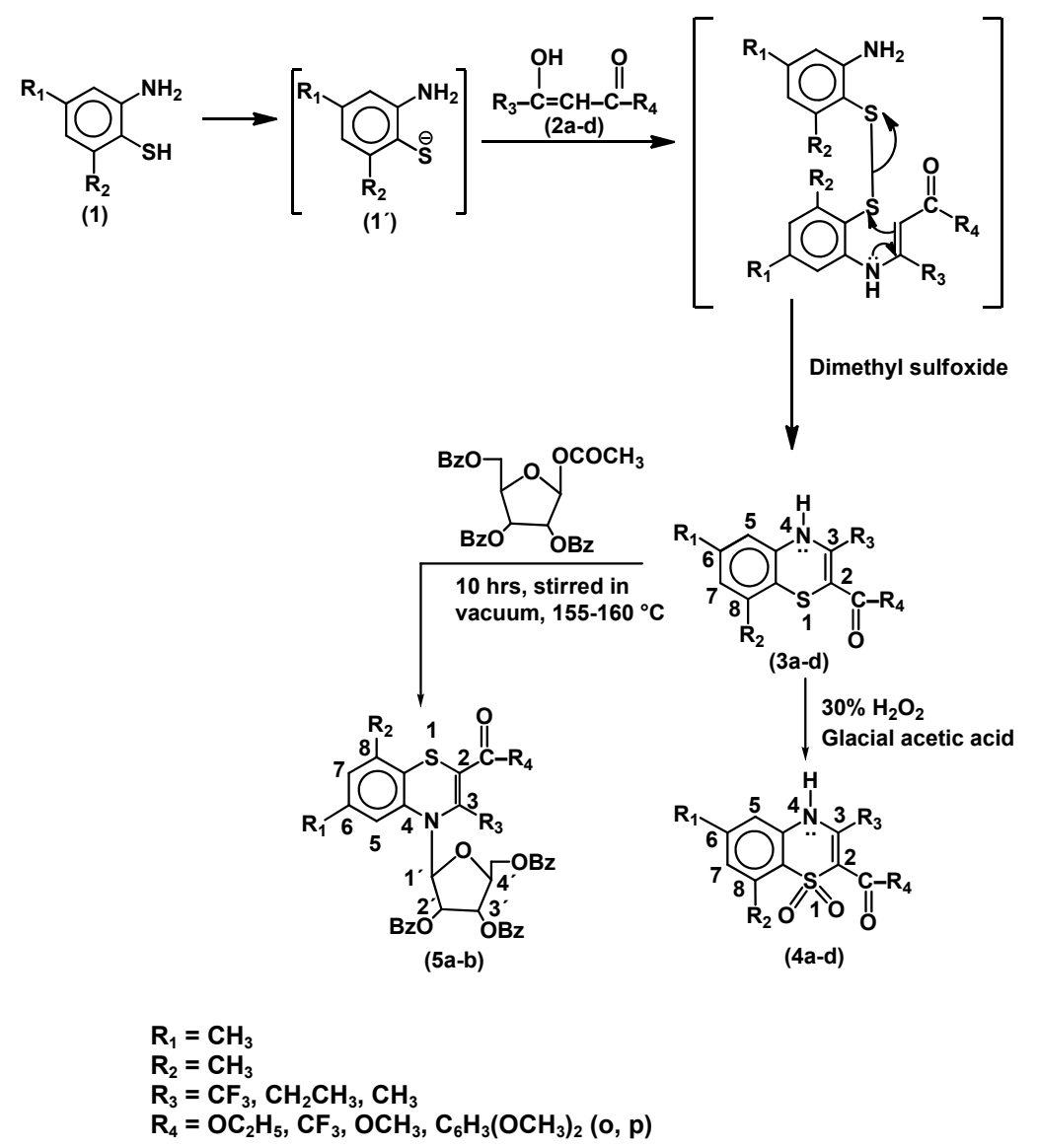

MS (m/z, \%): $341\left(\mathrm{M}^{+}\right), 244$ (58), 202 (29), 327 (78), 97 (100). Anal. calcd. for $\mathrm{C}_{13} \mathrm{H}_{9} \mathrm{NOF}_{6} \mathrm{~S}$ : C, 45.74; H, 2.63; N, 4.10. Found: C, 45.98; H, 2.65; N, 4.06\%.

Methyl-3-ethyl-6,8-dimethyl-4H-1,4-benzothiazine-2carboxylate (3c): Yield: $76 \%$. M.p.: $115^{\circ} \mathrm{C}$. IR $\left(\mathrm{KBr}, v, \mathrm{~cm}^{-1}\right)$ : 3280 (N-H), 1700 (>C=0), 1240, 1055 (C-O-C str.), $2875\left(-\mathrm{CH}_{3}\right.$ str.). ${ }^{1} \mathrm{H}$ NMR (DMSO- $\left.d_{6}, 300 \mathrm{MHz}, \delta, \mathrm{ppm}\right): 8.40(\mathrm{~s}, 1 \mathrm{H}, \mathrm{NH})$, 7.29-6.38 (m, 2H, aromatic- $\mathrm{H}), 2.35\left(\mathrm{~s}, 3 \mathrm{H},-\mathrm{CH}_{3}\right.$ at $\left.\mathrm{C}_{6}\right), 2.36(\mathrm{~s}$, $3 \mathrm{H},-\mathrm{CH}_{3}$ at $\left.\mathrm{C}_{8}\right), 2.00\left(\mathrm{q}, 2 \mathrm{H}, J=7.01 \mathrm{~Hz}, \mathrm{CH}_{2}\right.$ of $\mathrm{CH}_{2} \mathrm{CH}_{3}$ at $\left.\mathrm{C}_{3}\right)$, $1.06\left(\mathrm{t}, 3 \mathrm{H}, J=6 \mathrm{~Hz}, \mathrm{CH}_{3}\right.$ of $\mathrm{CH}_{2} \mathrm{CH}_{3}$ at $\left.\mathrm{C}_{3}\right), 3.76\left(\mathrm{~s}, 3 \mathrm{H},-\mathrm{CH}_{3}\right.$ of $\mathrm{OCH}_{3}$ at $\left.\mathrm{C}_{2}\right) .{ }^{13} \mathrm{C} \mathrm{NMR}\left(\mathrm{CDCl}_{3}, 300 \mathrm{MHz}, \delta, \mathrm{ppm}\right): 108.2(\mathrm{C}-2)$, 141.7 (C-3), 118.2 (C-5), 139.1 (C-6), 114.2 (C-7), 142.2 (C-8), $19.6\left(\mathrm{CH}_{3}\right.$ at $\left.\mathrm{C}_{6}\right), 14.8\left(\mathrm{CH}_{3}\right.$ at $\left.\mathrm{C}_{8}\right), 24.1\left(\mathrm{CH}_{2}\right.$ of $\mathrm{CH}_{2} \mathrm{CH}_{3}$ at $\left.\mathrm{C}_{3}\right), 8.5$ $\left(\mathrm{CH}_{3}\right.$ of $\mathrm{CH}_{2} \mathrm{CH}_{3}$ at $\mathrm{C}_{3}$ ), 165.0 (C of $\mathrm{CO}$ at $\mathrm{C}_{2}$ ), 50.1 ( $\mathrm{C}$ of $\mathrm{OCH}_{3}$ at
C2). MS (m/z, \%): $263\left(\mathrm{M}^{+}\right), 204$ (56), 162 (31), 221 (71), 59 (100). Anal. calcd. for $\mathrm{C}_{14} \mathrm{H}_{17} \mathrm{NO}_{2} \mathrm{~S}: \mathrm{C}, 63.87 ; \mathrm{H}, 6.46 ; \mathrm{N}, 5.32$. Found: C, 64.11; H, 6.40; N, 5.39\%.

2-(2',4'-Dimethoxybenzoyl)-3,6,8-trimethyl-4H-1,4-benzothiazine (3d): Yield: 80\%. M.p.: $53^{\circ} \mathrm{C}$. IR (KBr, $\left.v, \mathrm{~cm}^{-1}\right): 3298(\mathrm{~N}-$ $\mathrm{H}$ ), 1690 (>C=0), 1260, 1060 (C-O-C str.), $2886\left(\mathrm{CH}_{3}\right.$ str.). ${ }^{1} \mathrm{H}$ NMR (DMSO- $\left.d_{6}, 300 \mathrm{MHz}, \delta, \mathrm{ppm}\right): 9.09$ (s, 1H, NH), 7.16-6.08 $(\mathrm{m}, 2 \mathrm{H}$, aromatic- $\mathrm{H}), 1.71\left(\mathrm{~s}, 3 \mathrm{H},-\mathrm{CH}_{3}\right.$ at $\left.\mathrm{C}_{3}\right), 3.73\left(\mathrm{~s}, 3 \mathrm{H},-\mathrm{OCH}_{3}\right.$ at ortho position of $-\mathrm{COC}_{6} \mathrm{H}_{3}\left(\mathrm{OCH}_{3}\right)_{2}(\mathrm{o}, \mathrm{p})$ at $\left.\mathrm{C}_{2}\right), 3.78(\mathrm{~s}, 3 \mathrm{H}$, $\mathrm{OCH}_{3}$ at para position of $-\mathrm{COC}_{6} \mathrm{H}_{3}\left(\mathrm{OCH}_{3}\right)_{2}(\mathrm{o}, \mathrm{p})$ at $\left.\mathrm{C}_{2}\right), 2.14(\mathrm{~s}$, $3 \mathrm{H}, \mathrm{CH}_{3}$ at $\left.\mathrm{C}_{6}\right), 2.20\left(\mathrm{~s}, 3 \mathrm{H}, \mathrm{CH}_{3}\right.$ at $\left.\mathrm{C}_{8}\right), 7.20-5.95(\mathrm{~m}, 3 \mathrm{H}$, aromatic- $\mathrm{H}$ of benzoyl group). ${ }^{13} \mathrm{C} \mathrm{NMR}\left(\mathrm{CDCl}_{3}, 300 \mathrm{MHz}, \delta\right.$, ppm): 112.9 (C-2), 138.6 (C-3), 111.9 (C-5), 136.2 (C-6), 118.2 (C-7), 146.2 (C-8), $16.5\left(\mathrm{CH}_{3}\right.$ at $\left.\mathrm{C}_{3}\right), 187$ (C of $\mathrm{CO}$ at $\mathrm{C}_{2}$ ), $56.8(\mathrm{C}$ 
of $\mathrm{OCH}_{3}$ at ortho position $-\mathrm{COC}_{6} \mathrm{H}_{3}\left(\mathrm{OCH}_{3}\right)_{2}(\mathrm{o}, \mathrm{p})$ at $\left.\mathrm{C}_{2}\right)$. MS ( $\mathrm{m} / \mathrm{z}, \%)$ ) $355\left(\mathrm{M}^{+}\right), 190$ (61), 137 (48), 148 (28), 313 (70), 165 (100). Anal. calcd. for $\mathrm{C}_{20} \mathrm{H}_{21} \mathrm{NO}_{3} \mathrm{~S}$ : C, 67.60; H, 5.91; N, 3.94 . Found: C, 67.88; H, 5.89; N, 3.89\%.

\subsection{General method of synthesis of 4H-1,4-benzothiazine, 1,1-dioxides (sulfones) (4a-d)}

$30 \%$ Hydrogen peroxide ( $5 \mathrm{~mL}$ ) was added to a solution of 0.01 mole of $4 H$-1,4-benzothiazine in $20 \mathrm{~mL}$ glacial acetic acid and refluxed for 15 minutes at $50-55{ }^{\circ} \mathrm{C}$. Heating was stopped and another lot of $5 \mathrm{~mL}$ of $30 \%$. Hydrogen peroxide was added. The reaction mixture was again refluxed for 4-5 hrs. The excess of solvent was removed by distillation under reduced pressure and the solution was poured into a beaker containing crushed ice. The yellow residue separated out was filtered and then crystallized from ethanol (Scheme 1). Characterization and spectral data of these compounds (4a-d) is given as:

Ethyl-3-trifluoromethyl-6,8-dimethyl-4H-1,4-benzothiazine2-carboxylate-1,1-dioxide (4a): Yield: $69 \%$. M.p.: $70{ }^{\circ} \mathrm{C}$. IR (KBr, $\left.v, \mathrm{~cm}^{-1}\right): 3270(\mathrm{~N}-\mathrm{H}), 1700(>\mathrm{C}=0), 1180,1140\left(\mathrm{SO}_{2}\right.$ sym. str.), 1080 (C-S str.). ${ }^{1} \mathrm{H}$ NMR (DMSO- $d_{6}, 300 \mathrm{MHz}, \delta, \mathrm{ppm}$ ): 8.95 (s, $1 \mathrm{H}, \mathrm{NH}), 8.06-7.30(\mathrm{~m}, 2 \mathrm{H}$, aromatic- $\mathrm{H}), 4.20(\mathrm{q}, J=6.95 \mathrm{~Hz}, 2 \mathrm{H}$, $\mathrm{CH}_{2}$ of $\mathrm{C}_{2} \mathrm{H}_{5}$ a $\left.\mathrm{C}_{2}\right), 1.29\left(\mathrm{t}, 3 \mathrm{H}, J=6.3 \mathrm{~Hz}, \mathrm{CH}_{3}\right.$ of $\mathrm{C}_{2} \mathrm{H}_{5}$ at $\left.\mathrm{C}_{2}\right), 2.35$ $\left(\mathrm{s}, 3 \mathrm{H},-\mathrm{CH}_{3}\right.$ at $\left.\mathrm{C}_{6}\right), 2.36\left(\mathrm{~s}, 3 \mathrm{H},-\mathrm{CH}_{3}\right.$ at $\left.\mathrm{C}_{8}\right) .{ }^{13} \mathrm{C}$ NMR $\left(\mathrm{CDCl}_{3}, 300\right.$

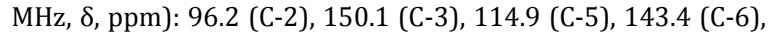
120.9 (C-7), 136.4 (C-8), $114.5\left(-\mathrm{CF}_{3}\right.$ at $\left.\mathrm{C}_{3}\right), 168$ (C of $\mathrm{CO}$ of $\mathrm{COOC}_{2} \mathrm{H}_{5}$ at $\left.\mathrm{C}_{2}\right), 59.6\left(\mathrm{CH}_{2}\right.$ of $\mathrm{COOC}_{2} \mathrm{H}_{5}$ at $\left.\mathrm{C}_{2}\right), 13.7\left(\mathrm{CH}_{3}\right.$ of $\mathrm{COOC}_{2} \mathrm{H}_{5}$ at $\left.\mathrm{C}_{2}\right)$. MS $(\mathrm{m} / \mathrm{z}, \%): 349\left(\mathrm{M}^{+}\right), 276(51), 234(29), 307$ (75), 73 (100). Anal. calcd. for $\mathrm{C}_{14} \mathrm{H}_{14} \mathrm{NO}_{4} \mathrm{~F}_{3} \mathrm{~S}$ : C, 48.13; H, 4.01; N, 4.01. Found: C, 48.35; H, 4.05; N, 4.03\%.

2-Trifluoroaceyl-3-trifluoromethyl-6,8-dimethyl-4H-1,4benzothiazine--1,1-dioxide (4b): Yield: $72 \%$. M.p.: $220^{\circ} \mathrm{C}$. IR $\left(\mathrm{KBr}, v, \mathrm{~cm}^{-1}\right): 3390(\mathrm{~N}-\mathrm{H}), 1680(>\mathrm{C}=0), 1172,1150\left(\mathrm{SO}_{2}\right.$ sym. str.), 1085 (C-S str.). ${ }^{1}$ H NMR (DMSO- $d_{6}, 300 \mathrm{MHz}, \delta, \mathrm{ppm}$ ): 8.30 (s, $1 \mathrm{H}, \mathrm{NH}), 8.16-7.08(\mathrm{~m}, 2 \mathrm{H}$, aromatic- $\mathrm{H}), 2.35\left(\mathrm{~s}, 3 \mathrm{H},-\mathrm{CH}_{3}\right.$ at $\left.\mathrm{C}_{6}\right), 2.36\left(\mathrm{~s}, 3 \mathrm{H},-\mathrm{CH}_{3}\right.$ at $\left.\mathrm{C}_{8}\right) .{ }^{13} \mathrm{C}$ NMR $\left(\mathrm{CDCl}_{3}, 300 \mathrm{MHz}, \delta, \mathrm{ppm}\right):$ 101 (C-2), 154 (C-3), 118 (C-5), 144 (C-6), 121 (C-7), 146 (C-8), $21.2\left(\mathrm{CH}_{3}\right.$ at $\left.\mathrm{C}_{6}\right), 13.7\left(-\mathrm{CH}_{3}\right.$ at $\left.\mathrm{C}_{3}\right), 114\left(-\mathrm{CF}_{3}\right.$ at $\left.\mathrm{C}_{3}\right), 196.8$ (C of $\mathrm{CO}$ at $\left.\mathrm{C}_{2}\right), 128.8\left(\mathrm{CF}_{3}\right.$ at $\left.\mathrm{C}_{2}\right)$. MS $(\mathrm{m} / \mathrm{z}, \%): 373\left(\mathrm{M}^{+}\right), 276(48)$, 234 (28), 331 (72), 97 (100). Anal. calcd. for $\mathrm{C}_{13} \mathrm{H}_{9} \mathrm{NO}_{3} \mathrm{~F}_{6} \mathrm{~S}$ : C, 41.82; H, 2.41; N, 3.75. Found: C, 42.09; H, 2.39; N, 3.78\%.

Methyl-3-Ethyl-6,8-dimethyl-4H-1,4-benzothiazine-2carboxylate-1,1-dioxide (4c): Yield: $56 \%$. M.p.: $240{ }^{\circ} \mathrm{C}$. IR (KBr, $\left.v, \mathrm{~cm}^{-1}\right): 3290(\mathrm{~N}-\mathrm{H}), 1710(>\mathrm{C}=0), 1165,1160$ ( $\mathrm{SO}_{2}$ sym. str.), 1095 (C-S str.). ${ }^{1} \mathrm{H}$ NMR (DMSO- $d_{6}, 300 \mathrm{MHz}, \delta, \mathrm{ppm}$ ): 8.80 (s, $1 \mathrm{H}, \mathrm{NH}), 7.30-6.40(\mathrm{~m}, 2 \mathrm{H}$, aromatic- $\mathrm{H}), 2.16(\mathrm{q}, 2 \mathrm{H}, J=7.2 \mathrm{~Hz}$, $\mathrm{CH}_{2}$ of $\mathrm{CH}_{2} \mathrm{CH}_{3}$ at $\left.\mathrm{C}_{3}\right), 1.09$ (t, $3 \mathrm{H}, J=6.4 \mathrm{~Hz}, \mathrm{CH}_{3}$ of $\mathrm{CH}_{2} \mathrm{CH}_{3}$ at $\left.\mathrm{C}_{3}\right), 3.79\left(\mathrm{~s}, 3 \mathrm{H},-\mathrm{CH}_{3}\right.$ of $\mathrm{OCH}_{3}$ at $\left.\mathrm{C}_{2}\right), 1.86\left(\mathrm{~s}, 3 \mathrm{H}, \mathrm{CH}_{3}\right.$ at $\left.\mathrm{C}_{6}\right), 2.31$ (s, $3 \mathrm{H}, \mathrm{CH}_{3}$ at $\left.\mathrm{C}_{8}\right) .{ }^{13} \mathrm{C} \mathrm{NMR}\left(\mathrm{CDCl}_{3}, 300 \mathrm{MHz}, \delta, \mathrm{ppm}\right): 99.2$ (C-2), 144 (C-3), 113.8 (C-5), 145.1 (C-6), 120.9 (C-7), 143 (C-8), 25.2 $\left(\mathrm{CH}_{2}\right.$ of $\mathrm{CH}_{2} \mathrm{CH}_{3}$ at $\left.\mathrm{C}_{3}\right), 8.9\left(\mathrm{CH}_{3}\right.$ at $\mathrm{CH}_{2} \mathrm{CH}_{3}$ at $\left.\mathrm{C}_{3}\right)$. $\mathrm{MS}(\mathrm{m} / \mathrm{z}, \%)$ : $295\left(\mathrm{M}^{+}\right), 236$ (38), 194 (30), 253 (76), 59 (100). Anal. calcd. for $\mathrm{C}_{14} \mathrm{H}_{17} \mathrm{NO}_{4} \mathrm{~S}$ : C, 56.94; H, 5.76; N, 4.74. Found: C, 57.22; H, $5.70 ; \mathrm{N}, 4.78 \%$.

2-(2',4'-Dimethoxybenzoyl)-3,6-8-trimethyl-4H-1,4-benzothiazine-1,1-dioxide (4d): Yield: $82 \%$. M.p.: $81^{\circ} \mathrm{C}$. IR (KBr, $v, \mathrm{~cm}-$ 1): $3320(\mathrm{~N}-\mathrm{H}), 1700$ (>C=0), 1177, 1150 ( $\mathrm{SO}_{2}$ sym. str.), 1077 (C-S str.). ${ }^{1} \mathrm{H}$ NMR (DMSO-d $6,300 \mathrm{MHz}, \delta, \mathrm{ppm}$ ): 9.12 (s, $1 \mathrm{H}$, $\mathrm{NH})$, 7.23-6.09 (m, 2H, aromatic- $\mathrm{H}), 1.92\left(\mathrm{~s}, 3 \mathrm{H},-\mathrm{CH}_{3}\right.$ at $\left.\mathrm{C}_{3}\right)$, $3.92\left(\mathrm{~s}, 3 \mathrm{H},-\mathrm{OCH}_{3}\right.$ at ortho position of $-\mathrm{COC}_{6} \mathrm{H}_{3}\left(\mathrm{OCH}_{3}\right)_{2}(\mathrm{o}, \mathrm{p})$ at $\left.\mathrm{C}_{2}\right), 3.79\left(\mathrm{~s}, 3 \mathrm{H},-\mathrm{OCH}_{3}\right.$ at para position of $\mathrm{COC}_{6} \mathrm{H}_{3}\left(\mathrm{OCH}_{3}\right)_{2}(\mathrm{o}, \mathrm{p})$ at $\mathrm{C}_{2}$ ). ${ }^{13} \mathrm{C}$ NMR $\left(\mathrm{CDCl}_{3}, 300 \mathrm{MHz}, \delta, \mathrm{ppm}\right): 97$ (C-2), 153 (C-3), 113.8 (C-5), 138 (C-6), 123 (C-7), 145 (C-8), $16.2\left(\mathrm{CH}_{3}\right.$ at $\left.\mathrm{C}_{3}\right)$, 189 ( $\mathrm{C}$ of $\mathrm{CO}$ of $\mathrm{COC}_{6} \mathrm{H}_{3}\left(\mathrm{OCH}_{3}\right)_{2}(\mathrm{o}, \mathrm{p})$ at $\left.\mathrm{C}_{2}\right), 56.9\left(\mathrm{C}\right.$ of $\mathrm{OCH}_{3}$ at ortho position $\mathrm{COC}_{6} \mathrm{H}_{3}\left(\mathrm{OCH}_{3}\right)_{2}(\mathrm{o}, \mathrm{p})$ at $\left.\mathrm{C}_{2}\right)$. MS $(\mathrm{m} / \mathrm{z}, \%): 387$ $\left(\mathrm{M}^{+}\right), 222$ (50), 180 (21), 345 (70), 165 (100). Anal. calcd. for $\mathrm{C}_{20} \mathrm{H}_{21} \mathrm{NO}_{5} \mathrm{~S}: \mathrm{C}, 62.01 ; \mathrm{H}, 5.42$; N, 3.61. Found: C, 62.26; H, 5.38; N, $3.56 \%$.

\subsection{General method of synthesis of substituted $N-\left(2^{\prime}, 3^{\prime}, 5^{\prime}-\right.$ tri-} o-benzoyl- $\beta$-D-ribofuranosyl)benzothiazine (5a-b)

To a concentrated solution of synthesized benzothiazines (3a-d), (0.002) mole in toluene, $\beta$-D-ribofuranose- 1 -acetate2,3,5-tribenzoate (0.002) mole was added and stirred, in vacuuo, on an oil bath, at $155-160{ }^{\circ} \mathrm{C}$, for 15 minutes. The vacuum was broken and the reaction was protected from moisture by using a guard tube. Stirring was further continued for 10-15 hours with application of vacuum for 15 minutes after every hour. The melt was dissolved in methanol, boiled for 10 minutes and cooled to room temperature. The precipitate was filtered and the filtrate was evaporated to dryness. The viscous residue, thus obtained was dissolved in ether, filtered, concentrated and kept in refrigerator overnight to get the crystalline compound (5a-b) (Scheme 1). Characterization and spectral data of these compounds (5a-b) is given as:

$N$-(2',3',5'-tri-O-benzoyl- $\beta$-D-ribofuranosyl)-ethyl-3-trifluoro methyl-6,8-dimethyl-4H-1,4-benzothiazine-2-carboxylate (5a): Yield: 84\%. M.p.: $80^{\circ} \mathrm{C}$. IR (KBr, $\left.v, \mathrm{~cm}^{-1}\right): 1700(\mathrm{C}=0), 1350$, 1170 (-CF3 str.), 1170 (C-O-C str.). ${ }^{1} \mathrm{H}$ NMR (DMSO- $d_{6}, 300 \mathrm{MHz}$, $\delta$, ppm): 8.52-7.10 (m, $2 \mathrm{H}$, aromatic- $\mathrm{H}), 4.21(\mathrm{q}, 2 \mathrm{H}, J=6.8 \mathrm{~Hz}$, $\mathrm{CH}_{2}$ of $\mathrm{C}_{2} \mathrm{H}_{5}$ at $\left.\mathrm{C}_{2}\right), 1.40\left(\mathrm{t}, 3 \mathrm{H}, J=6.86 \mathrm{~Hz},{ }^{\prime} \mathrm{CH}_{3}\right.$ of $\mathrm{C}_{2} \mathrm{H}_{5}$ at $\left.\mathrm{C}_{2}\right) .{ }^{13} \mathrm{C}$ NMR ( $\left.\mathrm{CDCl}_{3}, 300 \mathrm{MHz}, \delta, \mathrm{ppm}\right): 108.2$ (C-2), 139 (C-3), 113.2 (C-5), 136.8 (C-6), 121.2 (C-7), 140.2 (C-8), 66.8 (C-1'), 78.5 (C$\left.2^{\prime}\right), 72.5\left(\mathrm{C}-3^{\prime}\right), 71.6\left(\mathrm{C}-4^{\prime}\right)$. MS $(m / z, \%): 761\left(\mathrm{M}^{+}\right), 688$ (39), 646 (31), 73 (100). Anal. calcd. for $\mathrm{C}_{40} \mathrm{H}_{34} \mathrm{NO}_{9} \mathrm{~F}_{3} \mathrm{~S}$ : C, 63.07; $\mathrm{H}$, 4.46; N, 1.83. Found: C, 63.28; H, 4.40; N, 1.80\%. Optical rotation $[\alpha]_{\mathrm{D}} 23=-17.28^{\circ} \mathrm{C}$.

$N$ - $\left(2^{\prime}, 3^{\prime}, 5^{\prime}-\right.$ Tri-O-benzoyl- $\beta$-D-ribofuranosyl)-2-trifluoro acetyl-3-trifluoromethyl-6,8-dimethyl-4H-1,4-benzothiazine (5b): Yield: 68\%. M.p.: $82{ }^{\circ} \mathrm{C}$. IR (KBr, $\left.v, \mathrm{~cm}^{-1}\right)$ : 1680 (C=0), 1355, 1185 (-CF 3 str.), 1165 (C-O-C str.). ${ }^{1} \mathrm{H}$ NMR (DMSO-d $d_{6}, 300$ $\mathrm{MHz}, \delta, \mathrm{ppm}): 8.28-7.01(\mathrm{~m}, 2 \mathrm{H}$, aromatic- $\mathrm{H}), 2.38\left(\mathrm{~s}, 2 \mathrm{H}, \mathrm{CH}_{3}\right.$ at $\left.\mathrm{C}_{6}\right), 2.37$ (s, 3H, $-\mathrm{CH}_{3}$ at $\left.\mathrm{C}_{8}\right) .{ }^{13} \mathrm{C} \mathrm{NMR}\left(\mathrm{CDCl}_{3}, 300 \mathrm{MHz}, \delta, \mathrm{ppm}\right)$ : 117.2 (C-2), 173.3 (C-3), 113.6 (C-5), 136.4 (C-6), 120 (C-7), 136 (C-8), 69.2 (C-1'), 75.2 (C-2'), 76.1 (C-3'), 72.1 (C-4'). MS $(\mathrm{m} / \mathrm{z}, \%): 785\left(\mathrm{M}^{+}\right), 688(37), 646$ (27), 97 (100). Anal. calcd. for $\mathrm{C}_{39} \mathrm{H}_{29} \mathrm{NO}_{8} \mathrm{~F}_{6} \mathrm{~S}$ : C, 59.61; H, 3.69; N, 1.78. Found: C, 59.83; H, 3.63; N, $1.76 \%$. Optical rotation $[\alpha]_{\mathrm{D}} 23=-20.18^{\circ} \mathrm{C}$.

\subsection{Biological activity}

\subsubsection{Antioxidant activity}

\subsubsection{DPPH radical scavenging assay}

Radical scavenging activity of the synthesized compounds against stable 1,1-diphenyl-2-picrylhydrazyl (DPPH) radical was determined spectrophotometrically as described by Cuendet et al. [12]. A stock solution of $1 \mathrm{mg} / \mathrm{mL}$ of the compound was prepared in methanol. $50 \mu \mathrm{L}$ of compounds were added to $5 \mathrm{~mL}$ of a $0.004 \%$ methanol solution of DPPH. After 30 minutes incubation in dark at room temperature, absorbance was read against a blank at $517 \mathrm{~nm}$ (Table 2). Since IC50 Value is inversely related to the antioxidant activity; ascorbic acid, which is a very good antioxidant, shows a lower IC50 value of $17.8 \mu \mathrm{g} / \mathrm{mL}$. Among all the synthesized compounds; compound 3c shows a value of $23.56 \mu \mathrm{g} / \mathrm{mL}$ which shows its good antioxidant nature.

The assay was carried out in triplicate and the percentage of inhibition was calculated by using the following formula.

$\%$ Inhibition $=\frac{(\mathrm{AB}-\mathrm{AA})}{\mathrm{AB}} \times 100$

where $\mathrm{AB}=$ Absorption of blank, $\mathrm{AA}=$ Absorption of test, Ascorbic acid (shows antioxidant activity) as a positive control and methanol (no antioxidant activity) as a negative control has been used in this assay. 
Table 2. Antioxidant activity of the synthesized compounds (DPPH) assay (3a-d, $\mathbf{4 a - d}, \mathbf{5 a}-\mathbf{b})$.

\begin{tabular}{|c|c|c|c|c|}
\hline Compound No & $\mathbf{R}_{3}$ & ( & DPPH \% inhibition of $1 \mathrm{mg} / \mathrm{mL}$ of compound & $\mathrm{IC}_{50}(\mu \mathrm{g} / \mathrm{mL})$ \\
\hline $3 a$ & $\mathrm{CF}_{3}$ & $\mathrm{OC}_{2} \mathrm{H}_{5}$ & $42.74 \pm 0.03$ & 823.78 \\
\hline $3 b$ & $\mathrm{CF}_{3}$ & $\mathrm{CF}_{3}$ & $65.13 \pm 0.02$ & 500.8 \\
\hline $3 c$ & $\mathrm{CH}_{2} \mathrm{CH}_{3}$ & $\mathrm{OCH}_{3}$ & $90.76 \pm 0.07$ & 23.56 \\
\hline $3 d$ & $\mathrm{CH}_{3}$ & $\mathrm{C}_{6} \mathrm{H}_{3}\left(\mathrm{OCH}_{3}\right)_{2}(o, p)$ & $70.79 \pm 0.08$ & 79.12 \\
\hline $4 a$ & $\mathrm{CF}_{3}$ & $\mathrm{OC}_{2} \mathrm{H}_{5}$ & $23.48 \pm 0.09$ & - \\
\hline $4 b$ & $\mathrm{CF}_{3}$ & $\mathrm{CF}_{3}$ & $67.49 \pm 1.10$ & 432.90 \\
\hline $4 c$ & $\mathrm{CH}_{2} \mathrm{CH}_{3}$ & $\mathrm{OCH}_{3}$ & $11.88 \pm 0.05$ & - \\
\hline $4 d$ & $\mathrm{CH}_{3}$ & $\mathrm{C}_{6} \mathrm{H}_{3}\left(\mathrm{OCH}_{3}\right)_{2}(o, p)$ & $34.10 \pm 1.20$ & - \\
\hline $5 a$ & $\mathrm{CF}_{3}$ & $\mathrm{OC}_{2} \mathrm{H}_{5}$ & $73.27 \pm 0.06$ & 62.70 \\
\hline $5 b$ & $\mathrm{CF}_{3}$ & $\mathrm{CF}_{3}$ & $75.37 \pm 0.08$ & 58.90 \\
\hline Ascorbic acid & & & $92.96 \pm 00.9$ & 17.82 \\
\hline
\end{tabular}

Table 3. Antioxidant activity f the synthesized compounds* (ABTS•++ assay) (3a-d, 4a-d, 5a-b).

\begin{tabular}{|c|c|c|c|c|c|c|c|}
\hline \multirow{2}{*}{ Compound No } & \multirow{2}{*}{$\mathbf{R}_{3}$} & \multirow{2}{*}{$\mathbf{R}_{4}$} & \multicolumn{5}{|c|}{ ABTS $\cdot+$ activity at different intervals (min.) } \\
\hline & & & $\mathbf{0}$ & 1 & 2 & 4 & 6 \\
\hline$\overline{3 a}$ & $\mathrm{CF}_{3}$ & $\mathrm{OC}_{2} \mathrm{H}_{5}$ & 0.697 & 0.696 & 0.691 & 0.687 & 0.482 \\
\hline $3 b$ & $\mathrm{CF}_{3}$ & $\mathrm{CF}_{3}$ & 0.698 & 0.303 & 0.264 & 0.097 & 0.025 \\
\hline $3 c$ & $\mathrm{CH}_{2} \mathrm{CH}_{3}$ & $\mathrm{OCH}_{3}$ & 0.688 & 0.109 & 0.09 & 0.002 & 0.002 \\
\hline $3 d$ & $\mathrm{CH}_{3}$ & $\mathrm{C}_{6} \mathrm{H}_{3}\left(\mathrm{OCH}_{3}\right)_{2}(o, p)$ & 0.695 & 0.087 & 0.039 & 0.025 & 0.025 \\
\hline $4 a$ & $\mathrm{CF}_{3}$ & $\mathrm{OC}_{2} \mathrm{H}_{5}$ & 0.699 & 0.525 & 0.406 & 0.365 & 0.339 \\
\hline $4 b$ & $\mathrm{CF}_{3}$ & $\mathrm{CF}_{3}$ & 0.686 & 0.392 & 0.367 & 0.294 & 0.262 \\
\hline $4 c$ & $\mathrm{CH}_{2} \mathrm{CH}_{3}$ & $\mathrm{OCH}_{3}$ & 0.693 & 0.682 & 0.609 & 0.382 & 0.329 \\
\hline $4 d$ & $\mathrm{CH}_{3}$ & $\mathrm{C}_{6} \mathrm{H}_{3}\left(\mathrm{OCH}_{3}\right)_{2}(o, p)$ & 0.700 & 0.276 & 0.162 & 0.027 & 0.021 \\
\hline $5 a$ & $\mathrm{CF}_{3}$ & $\mathrm{OC}_{2} \mathrm{H}_{5}$ & 0.686 & 0.194 & 0.095 & 0.032 & 0.016 \\
\hline $5 b$ & $\mathrm{CF}_{3}$ & $\mathrm{CF}_{3}$ & 0.714 & 0.228 & 0.195 & 0.069 & 0.023 \\
\hline Ascorbic acid & & & 0.694 & 0.040 & 0.003 & 0.003 & 0.003 \\
\hline
\end{tabular}

*Ascorbic acid is used as a reference compound.

Table 4. Antimicrobial activity of the synthesized compounds (3a-d, 4a-d, 5a-b).

\begin{tabular}{|c|c|c|c|c|c|c|}
\hline \multirow[b]{2}{*}{ Compound No } & \multirow[b]{2}{*}{$\mathbf{R}_{3}$} & \multirow[b]{2}{*}{$\mathbf{R}_{4}$} & \multicolumn{3}{|c|}{ Antibacterial activity a } & \multirow{2}{*}{$\begin{array}{l}\text { Antifungal activity } \\
\text { Candida albicans }\end{array}$} \\
\hline & & & $\begin{array}{l}\text { Coagulase negative } \\
\text { staphylococci }\end{array}$ & $\begin{array}{l}\text { Coagulase positive } \\
\text { staphylococci }\end{array}$ & Enterobacter & \\
\hline $3 a$ & $\mathrm{CF}_{3}$ & $\mathrm{OC}_{2} \mathrm{H}_{5}$ & 23 & 10 & 10 & 18 \\
\hline $3 \mathrm{~b}$ & $\mathrm{CF}_{3}$ & $\mathrm{CF}_{3}$ & 11 & 11 & 13 & 22 \\
\hline $3 c$ & $\mathrm{CH}_{2} \mathrm{CH}_{3}$ & $\mathrm{OCH}_{3}$ & 15 & 13 & - & 18 \\
\hline $3 d$ & $\mathrm{CH}_{3}$ & $\mathrm{C}_{6} \mathrm{H}_{3}\left(\mathrm{OCH}_{3}\right)_{2}(o, p)$ & 16 & 12 & 12 & 15 \\
\hline $4 a$ & $\mathrm{CF}_{3}$ & $\mathrm{OC}_{2} \mathrm{H}_{5}$ & 12 & 10 & - & - \\
\hline $4 \mathrm{~b}$ & $\mathrm{CF}_{3}$ & $\mathrm{CF}_{3}$ & 11 & 10 & 11 & 11 \\
\hline $4 c$ & $\mathrm{CH}_{2} \mathrm{CH}_{3}$ & $\mathrm{OCH}_{3}$ & - & - & - & 11 \\
\hline $4 d$ & $\mathrm{CH}_{3}$ & $\mathrm{C}_{6} \mathrm{H}_{3}\left(\mathrm{OCH}_{3}\right)_{2}(o, p)$ & 10 & 11 & - & 18 \\
\hline $5 a$ & $\mathrm{CF}_{3}$ & $\mathrm{OC}_{2} \mathrm{H}_{5}$ & 10 & - & 11 & 14 \\
\hline $5 b$ & $\mathrm{CF}_{3}$ & $\mathrm{CF}_{3}$ & 20 & 10 & - & 12 \\
\hline Gatifloxacin & & & - & - & 17 & - \\
\hline Vancomycin & & & 15 & 15 & - & - \\
\hline Flucanazole & & & - & - & - & 25 \\
\hline
\end{tabular}

a Zone of inhibition in $\mathrm{mm} ;<7 \mathrm{~mm}$ inactive; $7-9 \mathrm{~mm}$ weakly active; $10-12 \mathrm{~mm}$, moderately active; $>12 \mathrm{~mm}$, active.

b Zone of inhibition in $\mathrm{mm} ;<7 \mathrm{~mm}$ inactive; 7-11 mm weakly active; $12-17 \mathrm{~mm}$, moderately active; $>17 \mathrm{~mm}$, active.

\subsubsection{ABTS radical cation decolorization assay}

The 2,2-azinobis(3-ethybenzothiazoline-6-sulphonic acid) radical cation (ABTS*+) decolorization test was carried out using an improved assay of Re et al. [13]. In brief, ABTS*+ was generated by oxidation of ABTS with potassium persulphate. For this purpose, ABTS was dissolved in ionized water at concentration of $7 \mathrm{mM}$, and potassium persulphate was added to a concentration of $2.45 \mathrm{mM}$. The reaction mixture was left at room temperature overnight (12-15 hours), in the dark before use; the ABTS solution then was diluted with ethanol to an absorbance of $0.700 \pm 0.020$ at $734 \mathrm{~nm}$. After addition of $1 \mathrm{ml}$ of the diluted ABTS solution to $10 \mu \mathrm{L}$ of compound and mixing, absorbance readings were taken at $30^{\circ} \mathrm{C}$ at intervals of exactly 1-6 min. The experiment was carried out in triplicate (Table 3 and Figure 1). We have used ascorbic acid as a positive control and ethanol as a negative control in this assay.

\subsubsection{Antimicrobial activity}

The antimicrobial assay of the synthesized compounds was carried out by using paper disc method of Gould et al. [13] against some bacteria and fungi at $100 \mu \mathrm{g}$ per disc concentration using Vancomycin, Gatifloxacin as reference compounds against bacteria (Coagulase negative Staphylococci, Coagulase positive Staphylococci, Enterobacter) and Flucanazole against fungus (Candida albicans). Paper disc method includes preparation of plates by pouring molten media into sterile petriplates which was then allowed to solidify for 5 minutes and $0.1 \%$ inoculum suspension was swabbed uniformly and allowed to dry for $5 \mathrm{~min}$. The compound discs prepared were then placed over the plates and incubated for $37^{\circ} \mathrm{C}$ for $24 \mathrm{hrs}$. At the end inhibition zones were measured with ruler in millimetre. These microorganisms were obtained from Microbiology Department, Swai Man Singh Medical College, Jaipur (Table 4).

\section{Results and discussion}

2-Aminobenzenethiol (1) and $\beta$-diketones / $\beta$-ketoesters (2a-d) were refluxed in dimethyl sulfoxide which involves condensation and oxidative cyclization. A bis-(2-aminophenyl) disulfide (1') was obtained by oxidation of 2-aminobenzene thiol which cyclizes to form 4H-1,4-benzothiazines (3a-d) by cleavage of sulfur-sulfur bond due to high reactivity of $\alpha$ position of enaminoketone system towards nucleophillic attack. Compound (3a-d) on treatment with $30 \%$ hydrogen peroxide in glacial acetic acid were converted into their corresponding sulfones (4a-d). Treatment of (3a-b) in toluene with $\beta$-D-ribofuranose-1-acetate-2,3,5-tribenzoate in vacuum gave the corresponding ribofuranosides (5a-b) (Scheme 1). The structures of synthesized compounds are well supported by spectral data and elemental analysis. 


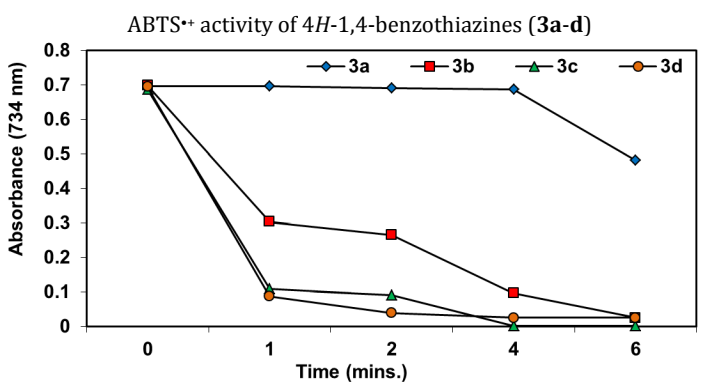

(a)

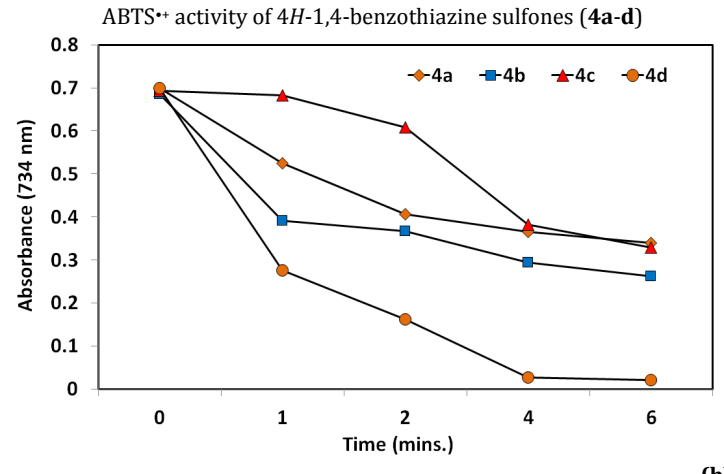

(b)

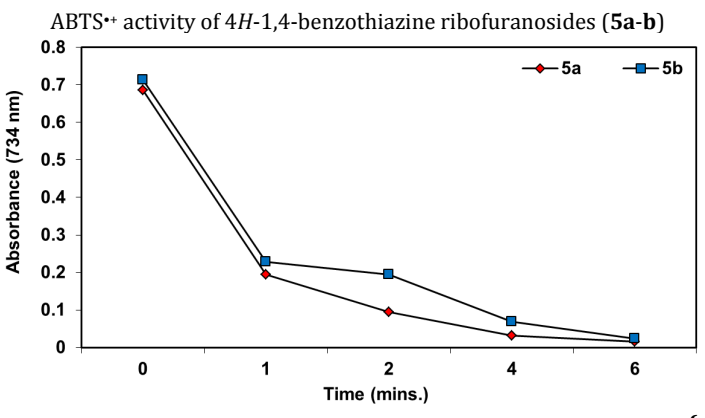

(c)

Figure 1. The effect of time on the suppression of absorbance of ABTS by synthesized compounds. After addition of $1 \mathrm{~mL}$ of diluted ABTS solution (A $734 \mathrm{~nm}=0.700 \pm 0.020$ ) to $10 \mu \mathrm{L}$ of the compound the absorbance reading was taken at $30^{\circ} \mathrm{C}$ exactly $1 \mathrm{~min}$., after initial mixing and up to 6 $\mathrm{min}$. All determinations were carried out in triplicates.

\subsection{Spectral analysis}

\subsubsection{IR spectra}

Compounds (3a-d) showed peaks in region 3385-3260 $\mathrm{cm}^{-1}$ due to $\mathrm{N}-\mathrm{H}$ stretching vibrations and $1700-1650 \mathrm{~cm}^{-1}$ due to $>\mathrm{C}=\mathrm{O}$ stretching vibrations which gets shifted to higher frequencies to $3390-3270 \mathrm{~cm}^{-1}$ and $1710-1680 \mathrm{~cm}^{-1}$, respectively, in compounds (4a-d). Compounds (4a-d) also exhibited two intense peaks in region $1360-1340 \mathrm{~cm}^{-1}$ and $1180-1140 \mathrm{~cm}^{-1}$ due to asymmetric and symmetric stretching vibrations of sulfonyl group. Compounds (4a-d) also showed C$\mathrm{S}$ stretching vibrations in region $1095-1077 \mathrm{~cm}^{-1}$.

Absence of stretching vibrations due to $>\mathrm{N}-\mathrm{H}$ group in compounds (5a-b) showed site of ribosylation, further in compounds (5a-b) bands due to $\mathrm{C}-\mathrm{O}-\mathrm{C}$ linkage of sugar appeared in the region $1170-1165 \mathrm{~cm}^{-1}$.

\subsection{2. ${ }^{1}$ H NMR spectra}

All compounds showed multiplet in region $\delta 8.52-6.06 \mathrm{ppm}$ due to aromatic protons and compounds (3a-d) and (4a-d) also showed a singlet due to $\mathrm{N}-\mathrm{H}$ proton in the region $\delta 9.12-8.29$ ppm. Peak due to $\mathrm{N}-\mathrm{H}$ proton was found to be absent in compounds (5a-b) due to ribosylation. In ribofuranosides, $\mathrm{C}_{4}^{\prime}{ }_{4}^{-}$ $\mathrm{H}$ proton showed multiplet in region $\delta 4.43-4.80 \mathrm{ppm}, \mathrm{C}_{2}{ }^{\prime}-\mathrm{H}$ and $\mathrm{C}_{3}{ }^{\prime}-\mathrm{H}$ protons appeared in region $\delta 4.51-5.90 \mathrm{ppm}$ as multiplet and $\mathrm{C}_{1}^{\prime}-\mathrm{H}$ proton appeared as doublet at $\delta 6.30-6.40$ ppm.

\subsubsection{Mass spectra}

The molecular ion peaks of 4H-1,4-benzothiazines were in accordance with their molecular weights. In all the cases side chain at $\mathrm{C}_{2}$ appears as a base peak which is obtained by its fission (Scheme 2).<smiles>[R]C(=O)C1=C([R3])N([R3])c2cc([R])cc([R2])c2SC1C([R])=O</smiles>

Scheme 2

\subsection{Biological activity (Antioxidant and Antimicrobial)}

All the synthesized compounds (3a-d), (4a-d), and (5a-b) were screened for their antioxidant activity by DPPH radical scavenging assay and (ABTS*+) radical cation decolorization assay. The synthesized compounds were also screened for antimicrobial activity (antibacterial and antifungal) by paper disc method. The present study demonstrated that these compounds showed mixed activity in DPPH and ABTS ${ }^{*+}$ assay.

(1) Compounds (3b, 3c, 3d, 4b, 5a, 5b) showed strong radical scavenging activity in DPPH assay that have DPPH \% inhibition $\geq 50$.

(2) Compounds (3a, 4d) showed moderate activity in DPPH assay that have DPPH \% inhibition $>30$.

(3) Compounds (4a, 4c) showed mild activity $(<30)$ in DPPH assay.

(4) Compounds (3b, 3c, 3d, $\mathbf{4 b}, \mathbf{4 d}, \mathbf{5 a}, \mathbf{5 b}$ ) were active in $\mathrm{ABTS}^{\bullet+}$ assay.

All these compounds were found to be moderately active against various bacteria such as (Coagulase negative Staphylococci, Coagulase positive Staphylococci, Enterobacter) and fungi (Candida albicans). Compounds (3a, 3c, 3d, 5b) showed good activity against Coagulase negative Staphylococci and compounds (3a, 3b, 3c and $\mathbf{4 d}$ ) shows good activity against Candida albicans.

\section{Conclusion}

The structures of synthesized compounds are well supported by spectral data and elemental analysis. The synthesized compounds were also screened for antioxidant activity (DPPH assay and ABTS•+ assay) and antimicrobial activity (antibacterial and antifungal). The present study demonstrated that these compounds showed mixed activity in DPPH and ABTS*+ assay. Compounds 3c, 3d showed excellent antioxidant activity in DPPH assay. Compounds $\mathbf{5 a}, \mathbf{5 b}$ showed much better activity in DPPH assay than compounds $\mathbf{3 a}, \mathbf{3 b}$ which are the precursors of $\mathbf{5 a}$ and $\mathbf{5 b}$. It shows that the ribofuranosides $\mathbf{5 a}$ and $\mathbf{5 b}$ showed much better antioxidant activity than their phenothiazine bases $(\mathbf{3 a}, \mathbf{3 b})$. This is due to the replacement of $\mathrm{H}$ by the sugar moiety (ribosylation). Compounds 3b, 3c, 3d, 4b, 5a, 5b showed good activity in both DPPH and ABTS assays.

The present paper is focused on the synthesis of novel heterocyclic compounds as possible antibacterial and antifungal agents. Compounds $\mathbf{3 c}$ and $\mathbf{3 d}$ showed antibacterial activity against Coagulase negative Staphylococci which is comparable to vancomycin. Compound $\mathbf{3 a}$ is much better than 
vancomycin in antibacterial activity against Coagulase negative Staphylococci. All these compounds were found to be moderately active against various bacteria such as (Coagulase negative Staphylococci, Coagulase positive Staphylococci, Enterobacter) and fungi (Candida albicans). Compounds (3a, 3c, 3d, 5b) showed good activity against Coagulase negative Staphylococci and compounds (3a, 3b, 3c and $\mathbf{4 d}$ ) shows good activity against Candida albicans.

\section{Acknowledgements}

The authors are grateful to Department of Chemistry, University of Rajasthan, Jaipur, India for providing necessary facilities. The University Grants Commission (Research Award Scheme) and Council of Scientific and Industrial Research, New Delhi, India are duly acknowledged for financial support. We are also thankful to Department of Zoology, University of Rajasthan and Sawai ManSingh Medical College, Jaipur, India for assistance in carrying out the biological activity.

\section{References}

[1]. Gautam, V.; Sharma, M.; Panwar, M.; Gautam, N.; Kumar, A.; Sharma, I. K.; Gautam, D. C. Phosphorus Sulfur 2009, 184(11), 3090-3109.

[2]. Gupta, S.; Ajmera, N.; Meena, P.; Gautam, N.; Kumar, A.; Gautam, D. C. Jordan J. Chem. 2009, 4(3), 209-221.

[3]. Gupta, V.; Gautam, R. K.; Jain, S. K.; Gupta, R. R. Phosphorus Sulfur 1990, 47(1) , 225-228.

[4]. Clercq, E. D. Nucleos. Nucleot. Nucl. 1985, 4(1), 3-11.

[5]. Gupta, A.; Saraswat, V.; Mukherji, S. K.; Gupta, R. R. Phosphorus Sulfur 1993, 85(1), 101-106.

[6]. Kachee, T. L.; Gupta, V.; Gautam, D. C.; Gupta, R. R. Phosphorus Sulfur 2005, 180(10), 2225-2234.

[7]. Gautam, N.; Hans, D.; Gautam, D. C. Oriental J. Chem. 2005, 21(2), 299302.

[8]. Kumar, N.; Singh, G.; Yadav, A. K. Heteroatom Chem. 2001, 12(1), 5256.

[9]. Gupta, R. R. Phenothiazines and 1,4-benzothiazines Chemical and Biomedical aspects, Elsevier, Amsterdam, 1988 pp. 160-210.

[10]. Sharma, P. R.; Gupta, V.; Gautam, D. C.; Gupta, R. R. Phosphorus Sulfur 2003, 178(7), 1483-1488.

[11]. Gautam, N.; Sharma, M.; Gautam, V. Asian J. Chem. 2010, 22(7), 53805388.

[12]. Cuendet, M.; Hostettmann, K.; Potterat, O.; Dyatmiko, W. Helv. Chim. Acta 1997, 80(4), 1144-1152.

[13]. Re, R.; Pellegrini, N.; Proteggente, A.; Pannala, A.; Yang, M.; Rice-Evans, C. Free Radic. Biol. Med. 1999, 26, 1231-1237.

[14]. Gould, J. C.; Browie, J. H. Edinb. Med. J. 1952, 59(4), 178-199. 